\title{
Tratamiento ortodoncico de un caso clinico de mal oclusion clase II - I
}

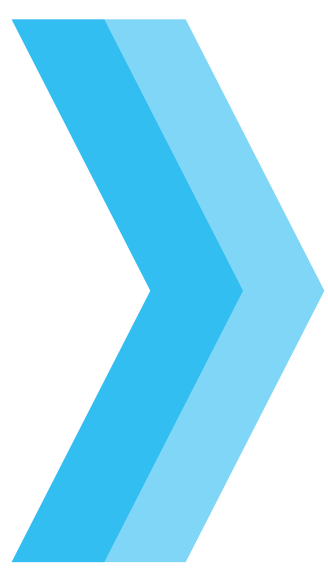

\section{Orthodontic treatment of a case of class II - I malocclusion}

\section{Yury Miguel Tenorio Cahuana ${ }^{1}$}

1. Cirujano Dentista. Profesor Auxiliar. Escuela de Odontología. Facultad de Ciencias de la Salud, UNJBG.

\section{RESUMEN}

Se reporta un caso de una paciente de 12 años y 6 meses de edad, con dentición permanente, dolicofacial, perfil convexo e hiperdivergente, presenta Patrón II con maloclusión clase II - 1 por retrusión mandibular, labio superior, labio inferior protruídos, mentón blando retruido, línea media desviada $1 \mathrm{~mm}$ izquierda mordida abierta anterior. Presenta además agenesia de las piezas dentarias 16 y 26, alteración de las funciones de la fonación, masticación, respiración. El tratamiento para este caso fue: ortodóncico.

\section{ABSTRACT}

We report a case of a patient of 12 years and 6 months of age, with permanent dentition, dolichofacial, convex and hyperdivergent profile, presents Pattern II with malocclusion class II -1 by mandibular retrusion, upper lip, protruded lower lip, soft chin retruded, middle line deviated $1 \mathrm{~mm}$ left anterior open bite. It also presents agenesis of teeth 16 and 26, alteration of the functions of phonation, chewing, breathing. The treatment for this case was: orthodontic. 


\section{INTRODUCCIÓN}

De acuerdo con la clasificación de Angle, se denominan Clases II, o distoclusiones las maloclusiones caracterizadas por la relación distal de la arcada dentaria inferior con respecto a la superior: tomando como referencia la cúspide mesiovestibular del primer molar maxilar, el surco del primer molar mandibular está situado por distal ${ }^{1}$.

La prevalencia de las maloclusiones clase II división 1 en la población europea es alrededor del $51 \%,{ }^{1}$ mientras en la población ecuatoriana en niños de 6 a 13 años es alrededor de $15.3 \%$ frente a un $77.3 \%$ de clase I, ${ }^{2}$ en la población colombiana es de $21 \%$ en cuanto a la relación de $75 \%$ de clase $1 .^{3}$ En cuanto a nuestra población los valores van de $11 \%$ al $16.25 \%$ a pesar que puede ser menor en los diferentes tipos de poblaciones. ${ }^{4}$

\section{Etiología}

Las maloclusiones son habitualmente variaciones clínicamente significativas de la fluctuación normal del crecimiento y morfología. Estas tienen dos causas básicas:

1) factores hereditarios o genéticos

2) factores ambientales, como trauma, agentes físicos, hábitos y enfermedades ${ }^{6}$.

Sin embargo es frecuente que sean el resultado de una compleja interacción entre varios factores que influyen en el crecimiento y el desarrollo y no siempre es posible describir un factor etiológico específico ${ }^{7}$.

Las maloclusiones según su etiología se dividen en:

a) Factores predisponentes:

- Existen factores hereditarios que se basan en el tamaño, forma de dientes, relación con el maxilar superior y mandíbula, función lingual y labial

- Existe la influencia de causas maternas y embrionarias que vienen a ser influencias prenatales.

b) Factores locales:

- Grupo intrínseco; se presenta la pérdida temprana de piezas temporales y permanentes, dientes supernumerarios, dientes que se pierden congénitamente, restauraciones que alteran a la oclusión normal.

- Factores circundantes o ambientales; anomalías a nivel de tejidos musculares periorales, hábitos.

- Factores sistémicos; enfermedades crónicas, mal funcionamiento del metabolismo y glándulas de secreción interna. ${ }^{2}$

De acuerdo a la filosofía MEAW:

- La maloclusión de clase II ángulo alto: el crecimiento antero-posterior de la maxila es pobre y la capacidad de la mandíbula para una adaptación anterior es insuficiente debido a la rotación posterior de la mandíbula relacionada al empinamiento del plano oclusal en el área molar.

- La maloclusión clase II ángulo bajo: es consecuencia de un déficit en la adaptación mandibular debido a una dimensión vertical insuficiente, soporte posterior disminuido, plano oclusal inclinado, no control retrusivo, interferencias posteriores. ${ }^{8,9}$

\section{Características clínicas ${ }^{10}$}

- Prognatismo maxilar y retrognatismo mandibular lo que da una convexidad facial.

- El labio superior sobresale con respecto al labio inferior.

- No haya sellado labial, manteniéndose la boca entre abierta cuando el paciente está en oclusión habitual.

- Al cerrar la boca aumenta la convexidad y protrusión labial debido a la contracción de la musculatura.

- Es característico observar que los incisivos superiores descansan sobre el labio inferior.

\section{Características radiográficas}

En una relación de Clase II puede deberse a una combinación de cuatro factores principales:

1. Exceso esquelético maxilar.

2. Exceso dental maxilar.

3. Deficiencia esquelética mandibular

4. Deficiencia dental mandibular. ${ }^{11}$

Rakosi propuso otra clasificación morfológica que considera cinco posibilidades:

1. Clase II dentoalveolar.

2. Posición anterior del complejo nasomaxilar.

3. Retrognatismo del maxilar inferior.

4. Clase II de causa neuromuscular.

5. Formas combinadas. ${ }^{12}$ 


\section{REPORTE DE CASO}

Paciente de sexo femenino de 12 años y 6 meses de edad, en ABEG y LOTEP, sin antecedentes médicos de importancia, antecedentes odontológicos: hermanos mayores con antecedentes de clase II

\section{Motivo de la consulta}

Quiere que le arreglen los dientes superiores

\section{Examen Clínico}

Análisis Extraoral: Mesocéfalo, mesofacial, perfil convexo e hiperdivergente, asimetría de tercios y quintos faciales, presenta Patrón II, no presenta buen sellado labial. (figura 1 y 2 )

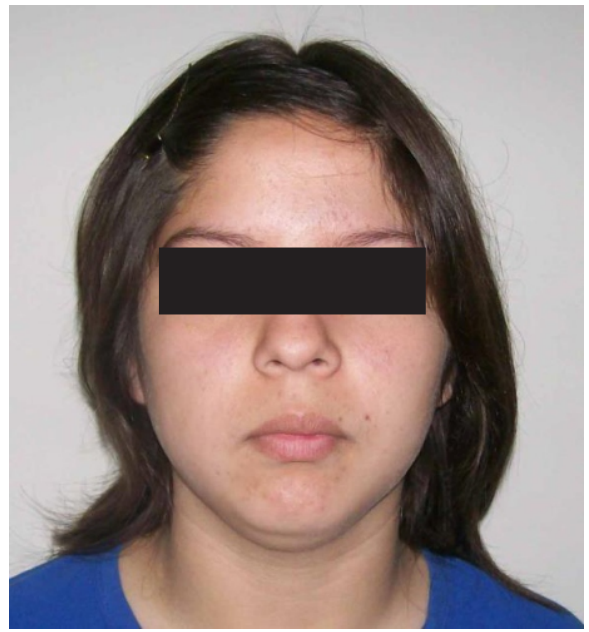

\section{Figura 1}

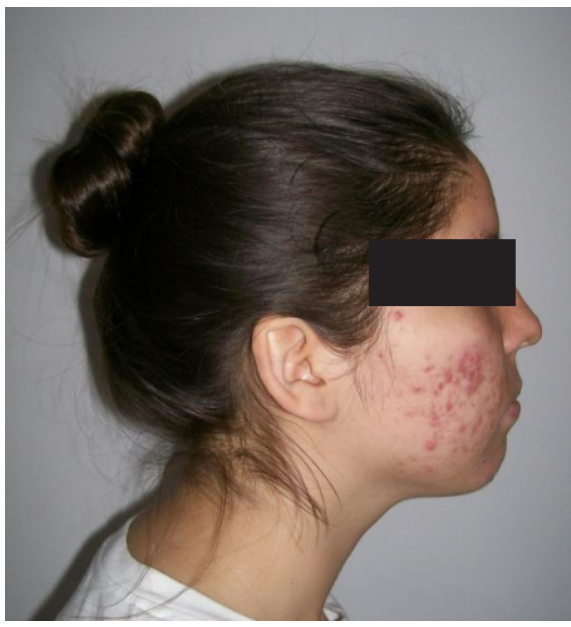

Figura 2

Análisis Intraoral: dentición permanente, con maloclusión clase II división 1 por retrusión mandibular, labio superior en posición normal, labio inferior ligeramente retruído y hipertrofia de la borla del mentón; RM y RC ambos lados clase II, OJ de $7 \mathrm{~mm}$, OB $3 \mathrm{~mm}$, línea media desviada $2 \mathrm{~mm}$ izquierda (inferior), arcadas superiore e inferior de forma triangular, mordida abierta anterior, discrepancia Maxilar Superior: $-7 \mathrm{~mm}$, Maxilar Inferior: $-4 \mathrm{~mm}$, Ausencia de Guía Anterior, alteración en las funciones de: fonación, masticación y respiración. (figuras 3, 4, 5, 6 y 7$)$

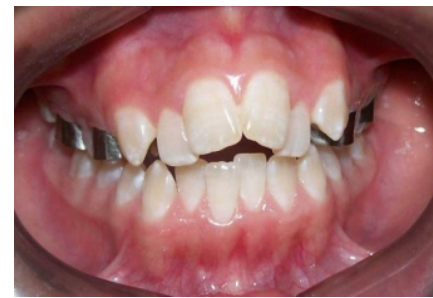

Figura 3

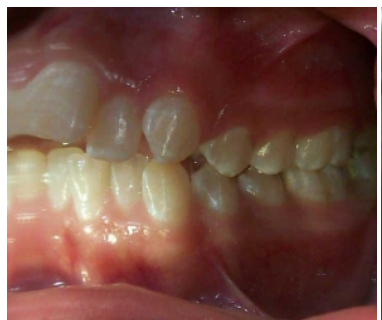

Figura 5

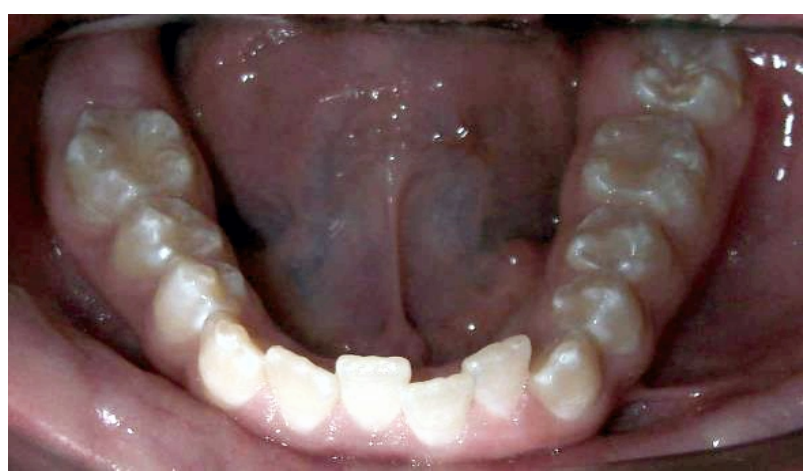

Figura 7

\section{Evaluación Radiográfica}

Análisis de la Radiografía Panorámica: se evidencia una dentición permanente, permeabilidad aérea obstruida (coana izquierda), cóndilos en su cavidad glenoidea, ausencia de los gérmenes dentarios de las piezas 18 y 28 (figuras 7 )

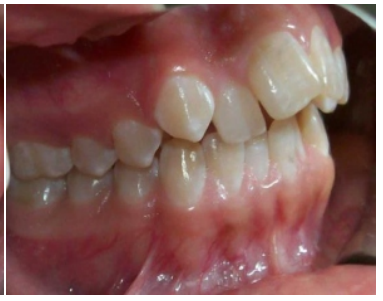

Figura 4

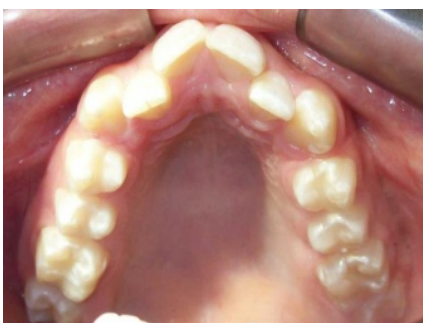

Figura 6

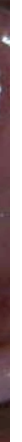

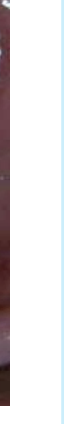

\section{(1)}

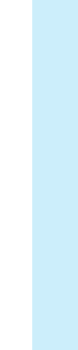




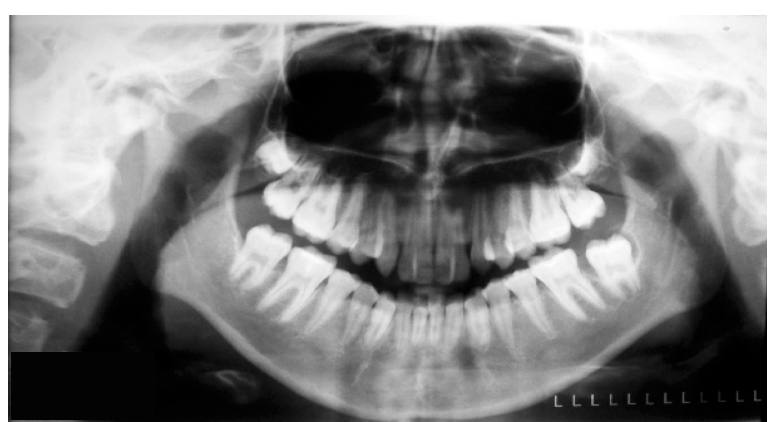

Figura 7

Análisis Cefalométrico: Paciente con relación esquelética clase II con retrusión mandibular, crecimiento horario, Incisivos superiores ligeramente vestibularizados y protruidos e incisivos inferiores lingualizados y retruídos. (figura 8)

En el análisis de la radiografía carpal se encontraba en el estadio fishmann de edad esquelética (13 años edad cronológica) (figura 9)

Análisis de modelos: Arco Superior de forma triangular, paladar profundo, Arco Inferior de forma triangular, curva de Spee moderada.

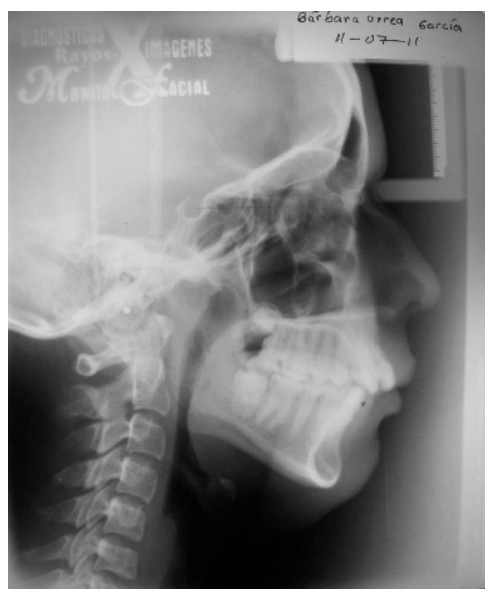

\begin{tabular}{|c|c|c|}
\hline Factor & V/Promedio & $11 / 7 / 11$ \\
\hline LBC & $70+/-2$ & $69 \mathrm{~mm}$ \\
\hline F.SN: & 80 & 7.50 \\
\hline SNA: & $82^{\circ}+/-2$ & $84^{\circ}$ \\
\hline SNB: & $800+/-2$ & 760 \\
\hline ANB: & $20+/-2$ & 8ㅇ \\
\hline Proj. USP & -3.0 a $-5.5 \mathrm{~mm}$ & $1.5 \mathrm{~mm}$ \\
\hline A-Nperp: & $0 \mathrm{a}+1 \mathrm{~mm}$ & $1.5 \mathrm{~mm}$ \\
\hline Pg-Nperp: & $-8 a-6 /-2 a+4$ & $-10 \mathrm{~mm}$ \\
\hline CoA: & $86 \mathrm{~mm}$ & $86 \mathrm{~mm}$ \\
\hline CoGn: & $107-110 \mathrm{~mm}$ & $110 \mathrm{~mm}$ \\
\hline AFAi: & $60-62 \mathrm{~mm}$ & 78 mm \\
\hline IND. VERT & & (Dolico leve) \\
\hline SN.MGo: & $32 \div+/-5$ & $42 \circ$ \\
\hline I-NA: & $4 \mathrm{~mm}$ & $5 \mathrm{~mm}$ \\
\hline I.NA: & 220 & 230 \\
\hline I-Orb & $6 \mathrm{~mm}$ & $-2 \mathrm{~mm}$ \\
\hline I-NB: & $4 \mathrm{~mm}$ & $7 \mathrm{~mm}$ \\
\hline I.NB: & 250 & $29 \stackrel{0}{-2}$ \\
\hline FMA & $>/=30^{\circ}$ & 300 \\
\hline FMIA & 850 & 530 \\
\hline IMPA & $870-900$ & 950 \\
\hline LS-Nper & $14+-8$ & $18^{\circ}$ \\
\hline H-NB & $9-11$ & 210 \\
\hline
\end{tabular}

Figura 8

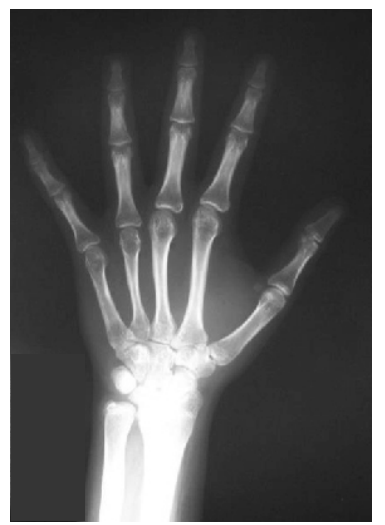

Figura 9

\section{Diagnóstico General}

Paciente de sexo femenino de 12 años y 6 meses de edad, mesofacial, Patrón II con relación esquelética clase II (retrusión mandibular), mal oclusión clase II división 1, Incisivos superiores ligeramente vestibularizados y protruidos e incisivos inferiores lingualizados y retruídos y discinesia de la borla del mentón con alteración de la fonación

\section{Objetivos de Tratamiento:}

- Derivar al otorrinolaringólogo (permeabilidad aérea)

- Corregir relación antero-posterior

- Corregir mordida mordida abierta anterior

- Mejorar relaciones dentarias

- Corregir la línea media

- Alinear e inclinar correctamente incisivos y caninos

- Corregir la proquelia y mentón

- Derivar al logopeda

\section{Tratamiento}

Se decide realizar primero un tratamiento en dos fases: Primero: Aplicación de un aparato de Hyrax para la disyunción de la sutura maxilar, provocando el aumento del diámetro transversal del arco superior, con activaciones de tornillo dos cuartos de vuelta al día durante 19 días para posteriormente dejar el aparato como contención mientras se continúa con la siguiente fase. (figura 10, 11, 12 y 13 )

Segundo: Aparato de ortodoncia con bandas y tubos dobles en las piezas dentarias: 16 y 26 , brackets: Roth, Slot $22 \times 28$. (figura 14 y 15 )

Luego se utilizó resorte para abrir espacio para las piezas 22,25 y 42 . (figuras $16,17,18$ y 19 ).

Se continuó con el tratamiento de ortodoncia; para el asentamiento de la mordida se utiliza elástico de 3/16 de pulgas en forma triangular y de caja. Se procede a retirar los brackets donde con el tratamiento se consiguen llaves molares y caninas de ambos lados Clase I así mismo la línea media interdentaria coincidente con la facial. (figuras $21,22,23,24$ y 25 )

Para la contención, se confeccionó un aparato de Hawley con arco vestibular modificado (acrílico). (figura 20) 


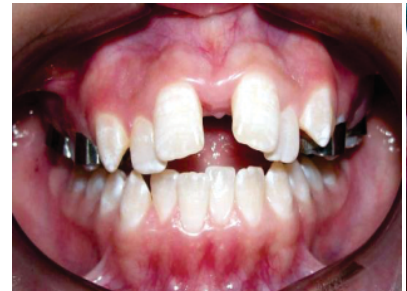

Figura 10

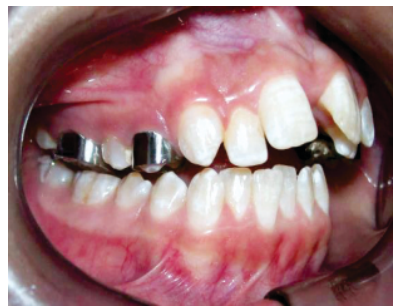

Figura 12

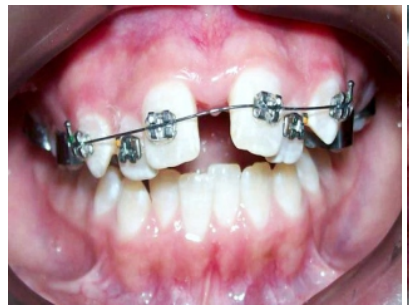

Figura 14

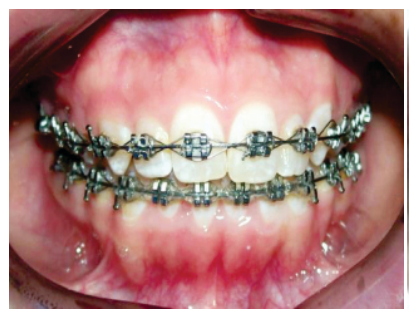

Figura 16

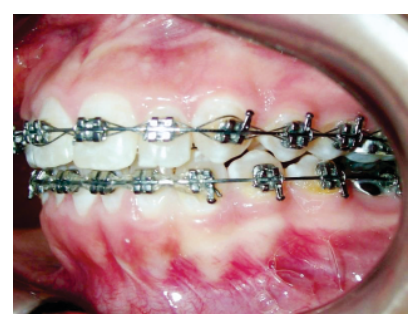

Figura 18

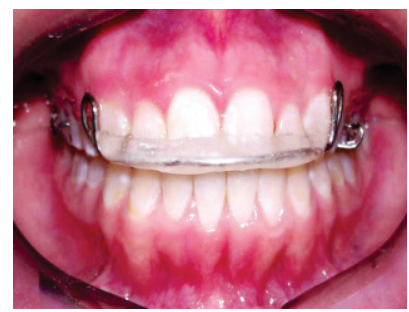

Figura 20

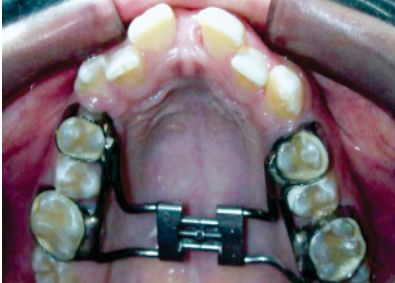

Figura 11

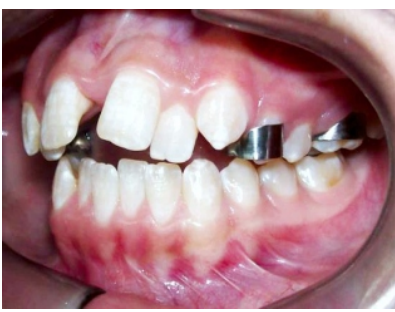

Figura 13

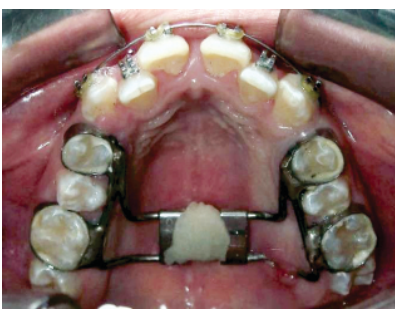

Figura 15

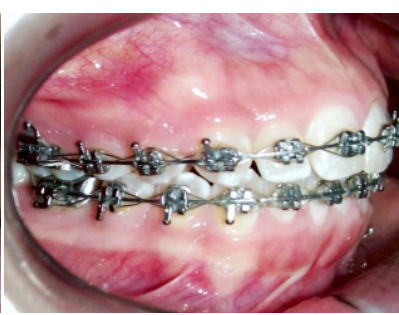

Figura 17

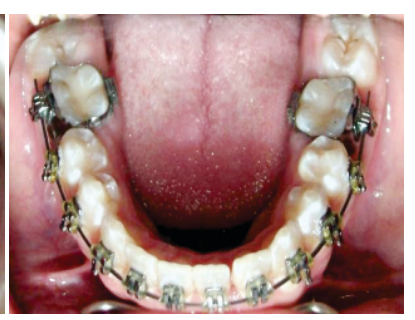

Figura 19

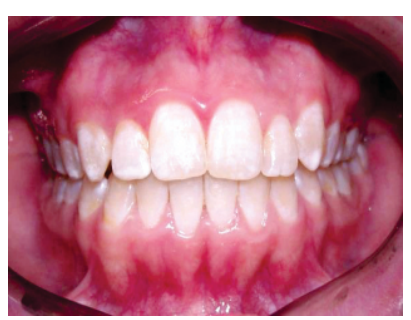

Figura 21
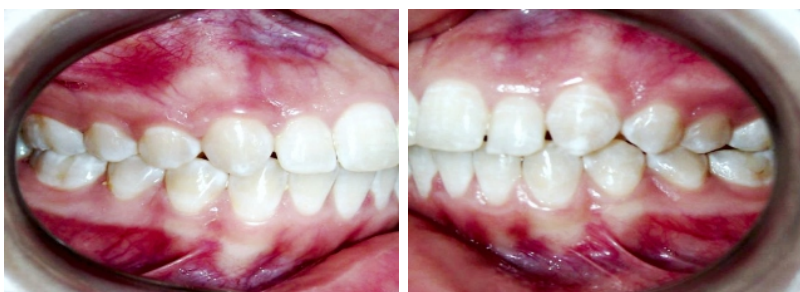

Figura 22

Figura 23

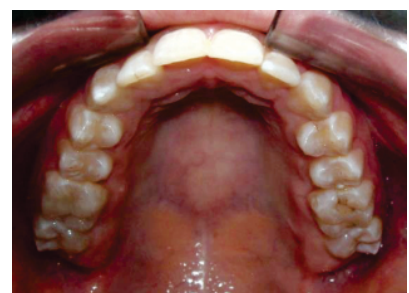

Figura 24

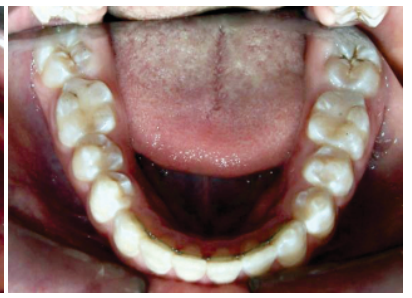

Figura 25

\section{DISCUSIÓN}

Los diversos abordajes terapéuticos para el tratamiento de las maloclusiones Clase II sugeridos en la literatura pueden ser, esencialmente dividas en tres grupos: modificación del crecimiento para reducir la discrepancia esquelética, camuflaje por movimiento dentario para compensación de la discrepancia esquelética, tratamiento ortodóncico - quirúrgico para reposicionamiento de los maxilares. ${ }^{13}$

Guest y col. Contrastaron los resultados del tratamiento de 50 pacientes de Clase II tratados con un disyuntor de acrílico adherido con los de 50 controles no tratados. El análisis de las cefalometrías seriadas tomadas con 4 años de diferencia indicaba que los aparatos de ERM adheridos ejerciían su mayor efecto a nivel oclusal, lo que producía específicamente una mejoría significativa de la relación molar de Clase II y una disminución del resalte. ${ }^{11}$

Para nuestro caso fue necesario recurrir a un tratamiento ortopédico, el cual fue necesario utilizar un aparato de Hyrax sin pistas lo cual nos permitió controlar el crecimiento vertical para luego proceder con la disyunción de la sutura palatina. Esta sobreexpación del maxilar, desajusta la oclusión provocando una adelantamiento de la mandíbula mejorando el resalte dental anterior.

Una vez terminada el tratamiento ortopédico se procedió con el tratamiento ortodóncico; con la colocación de brackets técnica Roth, específicamente en los cuatro incisivos superiores para ayudar a cerrar el diastema provocado por la disyunción. Continuando luego con el tratamiento ortodóncico de ambas arcadas. 
Se concluye, que tenemos que identificar es si esta maloclusión clase II tiene un componente dentario, alveolo dentario, esquelético, si es netamente funcional, si tiene antecedentes familiares, si hay un factor ambiental que la causado; o una combinación de ambas. Para luego planificar que tipo de tratamiento vamos a realizar. En nuestro caso es una maloclusión de clase II división 1 con retrusión mandibular, se le trató con una aparato de hyrax cuya disyunción provocó la liberalización de la mandíbula y su posterior adelantamiento así como un ligero desplazamiento hacia arriba del maxilar. Una terapia adecuada para este tipo de pacientes sin extracciones contribuye $a$ un mejor resultado oclusal, funcional y facial.

\section{REFERENCIAS BIBLIOGRÁFICAS}

1. Canut J. Ortodoncia clínica y terapéutica 2da edición 2005. Página 535

2. Andrade M. Prevalencia de la malocclusion dental en clínica de odontopediatría de la Universidad de las Américas en niños entre 6-13 años. UALA, Quito-Ecuador 2016. Página VI

3. Peña L, Gordillo de Mayorga Cl. Estudio epidemiológico de salud y malocclusion dental en niños de Bogotá. Colombia. Universidad Nacional de Colombia. Ed. El Malpensante. Bogotá, 2002.

4. Meneses A, Liñan C. Características cefalométricas en niños con malocclusion Clase II - 1 de 12 a 14 años de edad en ciudades con distinta altitud geográfica. Revista Herediana. Universidad Cayetano Heredia. Lima-Perú 2009. 19(2)

5. Viñas M, Muelas L. Estudio cefalométrico de la clase III. Universidad Complutense de Madrid, Madrid 2006, http:www.estudiocefalometricodelaclaseiii.htm.

6. Angle E. Treatment of malocclusion of the teeth and fractures of the maxillae, Angle's sistema. 6ta Edición. Philadelphia: SS White Dental Mfg Co; 1900.
7. Bishara S. Ortodoncia. Philadelphia: McGraw Hill; 2001.

8. Sadao S. Treatment approach to malocclusions under the consideration of craniofacial dynamics. Philippines: Grace Printing Press Inc.; 2001

9. Sadao S. MEAW, Orthodontic Therapy Using Multiloop Edgewise Archwire. kanagawa: Kanagawa Dental College; 2001.

10. Arriola $L$, Versatibilidad de la técnica Meaw en el tratamiento de las maloclusiones. Universidad Nacional Mayor de San Marcos. Lima - Perú 2012

11. Graber L, Vanarsdall R. Principios y técnicas actuales. Ortodoncia. 5ta. Edición Elsevier España 2012.

12. Rakosi T, Jonas I. Atlas de ortopedia maxilar: diagnóstico.Editorial Masson-Salvat. Barcelona 1992.

13. Pinho T, Figueiredo A. Orthodontic - orthognathic surgical treatment in a patient with class II subdivision malocclusion: occlusal plane alteration. American journal of orthodontics and dentofacial orthopedics 2011; 140(5): 703 - 12

\section{Correspondencia}

Yury Miguel Tenorio Cahuana

ymtc@yahoo.com
Fecha de recepción: 05 de octubre de 2017

Fecha de aceptación: 05 de noviembre de 2017 